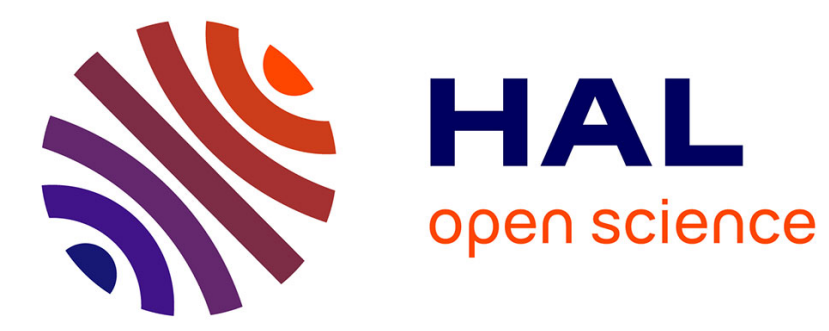

\title{
Optimization of tonal noise control with flow obstruction
}

\author{
Stéphane Moreau, Marlène Sanjose, Stéphan Magne
}

\section{To cite this version:}

Stéphane Moreau, Marlène Sanjose, Stéphan Magne. Optimization of tonal noise control with flow obstruction. 17th International Symposium on Transport Phenomena and Dynamics of Rotating Machinery (ISROMAC2017), Dec 2017, Maui, United States. hal-03004962

\section{HAL Id: hal-03004962 \\ https://hal.science/hal-03004962}

Submitted on 13 Nov 2020

HAL is a multi-disciplinary open access archive for the deposit and dissemination of scientific research documents, whether they are published or not. The documents may come from teaching and research institutions in France or abroad, or from public or private research centers.
L'archive ouverte pluridisciplinaire HAL, est destinée au dépôt et à la diffusion de documents scientifiques de niveau recherche, publiés ou non, émanant des établissements d'enseignement et de recherche français ou étrangers, des laboratoires publics ou privés. 


\title{
Optimization of tonal noise control with flow obstruction
}

\author{
Stéphane Moreau ${ }^{1 *}$, Marlène Sanjose ${ }^{1}$, Stéphan Magne ${ }^{1}$
}

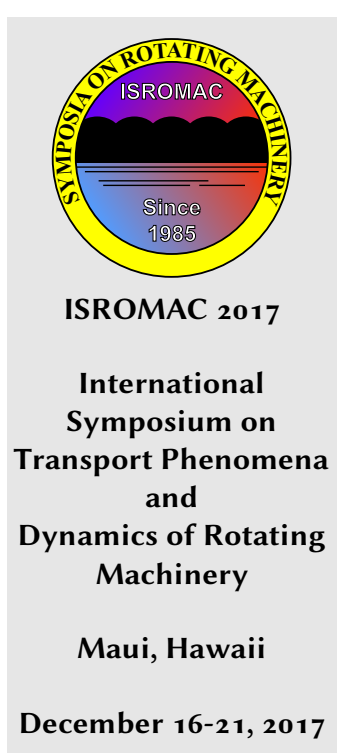

\begin{abstract}
The tonal noise control of an axial low-speed fan system with flow obstruction has been achieved both numerically and experimentally. Its primary noise source caused by rotor-wake interaction has been directly and accurately simulated with a Lattice Boltzmann Method. The latter has then allowed deciphering the noise control mechanism of an upstream sinusoidal obstruction: the vortex rings shed by the obstruction yield a second noise source at the rotor-blade leading edge. The obstruction itself does not create any significant noise and is acoustically transparent. An industrially-applicable numerical methodology has then been proposed to obtain the optimal obstruction design for a given fan geometry and operating condition, with a maximum of six simulations of the fan system without and with the obstruction being static and slowly rotating. Simulations with rotating obstructions provide the optimal lobe amplitude and an optimal obstruction angular position, which are found to be $20 \mathrm{~mm}$ and about $16^{\circ}$ respectively for the present fan system both numerically and experimentally. The frequency selectivity of the obstruction and the linear variation of the secondary source level with the lobe amplitude have also been confirmed.

\section{Keywords}

Aeroacoustics - Axial fan - ventilation noise - noise control

'Université de Sherbrooke, Département de Génie Mécanique, 2500 boulevard de l'Université, Sherbrooke, J1 $K_{2} R_{1}$, QC, Canada *Corresponding author: stephane.moreau@usherbrooke.ca
\end{abstract}

\section{INTRODUCTION}

The present study focuses on the tonal noise produced by low-speed axial fans that are often used in cooling and airconditioning systems. Such a noise component is not only a major part of the overall noise produced by such ventilation systems (about half or more depending on the operating condition), but also the major source of annoyance (subjective noise) when the tones emerge by more than $10 \mathrm{~dB}$ over the broadband level. In order to limit this nuisance, several passive and active noise control devices have been proposed $[1,2,3,4]$. A common feature seen for instance in automotive engine cooling fans is to modify the blade distribution to create an asymmetric fan with static balance that redistributes the acoustical energy at the blade passing frequency and its harmonics into more rotational harmonics. Yet, no complete control can be achieved and moreover the asymmetric blade distribution perturbs the turbulent flow field within the fan and consequently potentially increases the broadband noise component and the perceived overall sound. More recently Gerard et al. have proposed a passive-adaptive control of the tonal noise based on periodic obstructions placed upstream of the axial fan as shown in Fig. 1 (red sinusoidal obstruction with nine lobes), which can completely alleviate the tonal noise without modifying the overall sound and fan performances significantly $[5,6,7,8]$. Yet the exact noise control mechanism of this Simple Silence technology ${ }^{\mathrm{TM}}$ developed at Université de Sherbrooke (UdeS) is still controversial [9] and the optimization of the obstruction (shape and position) to achieve an optimal tonal noise
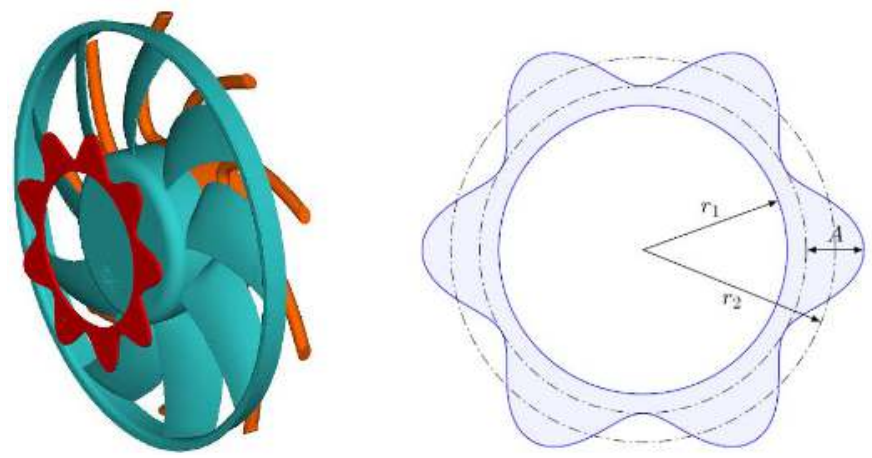

Figure 1. Controlled axial fan system with external obstruction.

control is still empirical and relies on several mock-up designs and lengthy experimental trial and error tests. Based on previous numerical simulations of tonal noise control of centrifugal fan systems [9, 10], an automatic and systematic numerical procedure is proposed here, which can bring both a better understanding of the noise mechanism control at stake, and a more efficient optimization procedure for the obstruction shape and position.

The control, experimental and numerical methodology is described in the next section. The numerical and experimental aerodynamic and acoustic results on the baseline and controlled axial fan system are compared in the following sections. Conclusions on the accuracy of the numerical method and the noise control strategy are finally drawn. 


\section{METHODOLOGY}

\subsection{Control strategy}

The methodology mostly relies on previous experimental and numerical results. Gerard et al. first showed that an additional inlet flow distortion induced by a carefully-shaped obstruction is able to significantly reduce tonal noise from rotorstator interaction (primary noise source) of low-speed fans having $B$ blades and spinning at a rotational speed $\Omega_{r}[11]$. They then studied several obstruction geometry and showed that the most harmonically selective was the sinusoidal geometry characterized by three parameters as shown in Fig. 1: the base radius $r_{1}$, the mid-lobe radius $r_{2}$, and the sinusoidal lobe amplitude $A[12,8]$. The optimized design for a given obstruction is then defined by two main parameters: $A_{\text {opt }}$, the radial amplitude of the obstruction which controls the magnitude of the obstruction-fan interaction (secondary noise source), and $\phi_{\text {opt }}$, the obstruction angular position which controls the phase of the secondary source. They also showed that the number of lobes $L$ can be chosen according to the harmonic order $n$ to be controlled, and that these obstructions are frequency selective: for each integer $n$, the geometrical parameters $\phi_{o p t}\left(n B \Omega_{r}\right)$ and $A_{o p t}\left(n B \Omega_{r}\right)$ mainly have an effect at the frequency $n B \Omega_{r}$. Moreover, around the optimal lobe amplitude $A_{\text {opt }}$, the secondary source level $20 \log _{10}\left(p_{s}\right)$ varies linearly with the lobe amplitude. They then found that the separation of the primary noise radiated by the fan system and the secondary noise induced by the flow obstruction could be separated by rotating the obstruction in the optimization process, which shifts the secondary tone (created by the obstruction-fan interaction) in the frequency spectrum [7]. All these assumptions are retained here and are checked a posteriori.

To achieve the present optimization the following additional assumptions are made. The obstruction-fan distance remains constant and the secondary source level is controlled by the lobe amplitude. It should be emphasized that the same methodology could be applied if the secondary source level was controlled by the obstruction-fan distance and the lobe amplitude remained constant. The obstruction-fan distance is also assumed to be larger than the maximum axial rotor chord in order to neglect the rotor potential effect on the obstruction. Finally, the obstruction rotational speed $\Omega_{\mathrm{o}}$ should be slow enough to assume a quasi-static flow at each angular position.

Based on the above assumptions the following control strategy is proposed and summarized in Fig. 2. Step 1 provides the reference configuration without the obstruction and characterizes the primary noise source to be controlled. It provides the primary acoustic field $p_{p}(\vec{x}, \omega)$ at an angular frequency $\omega$, and at the locations $\vec{x}$ where the noise should be controlled. The stationarity of this primary noise can be then evaluated by filtering the signal around the frequency $n B \Omega_{r}$ and plotting the temporal fluctuations of the Hilbert transform modulus. The stability of the Hilbert transform modulus appears to be a good indicator of the fan controllability. Step 2 estimates the optimal lobe amplitude of the

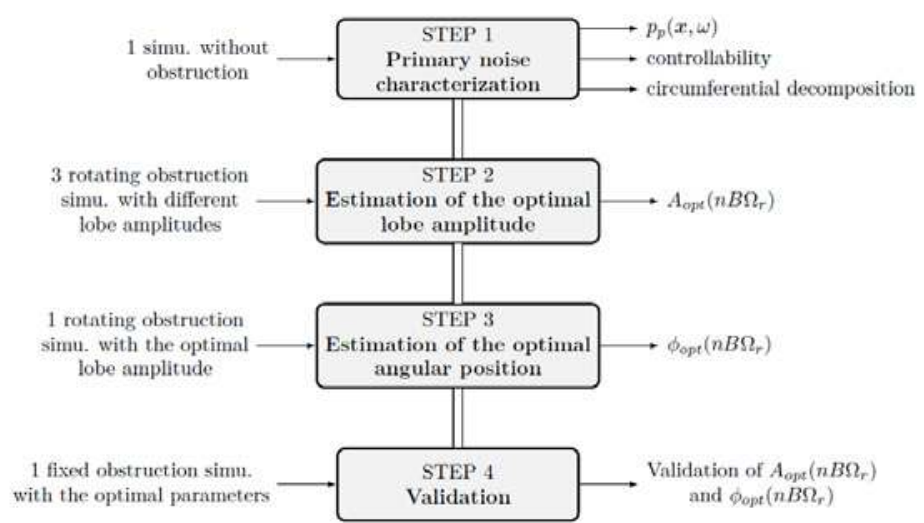

Figure 2. Overview of the methodology.

obstruction. A rotating obstruction is added to the baseline setup and three simulations are performed with different lobe amplitudes $A$. These computations may be run simultaneously. The obstruction rotates at the speed $\Omega_{\mathrm{o}}$ in the opposite direction of the rotor; hence it creates a secondary tone at the angular frequency $\omega=\mathrm{BPF}+\mathrm{LPF}=n B\left(\Omega_{r}+\Omega_{\mathrm{o}}\right)$, where $\mathrm{BPF}$ is the blade passing frequency and LPF is the lobe passing frequency. Since the tones of the primary and secondary sources are separated in the acoustic spectrum, the secondary source level $\log _{10} \mid p_{s}\left(\vec{x}, n B\left(\Omega_{r}+\Omega_{\mathrm{o}}\right) \mid\right.$ can be extracted without filtering. The optimal lobe amplitude $A_{o p t}\left(n B \Omega_{r}\right)$ is obtained from a linear regression of the secondary source level as a function of the lobe amplitude, at the value corresponding to the primary noise level $\log _{10}\left|p_{p}\left(\vec{x}, n B \Omega_{r}\right)\right|$. Step 3 estimates the optimal angular position. It is based on the time fluctuation of the total amplitude level $\left|p_{t}\left(\vec{x}, n B \Omega_{r}\right)\right|$ which is the combination of the primary and secondary sources. To extract $\left|p_{t}\left(\vec{x}, n B \Omega_{r}\right)\right|$ as a function of the angular position $\phi$, the time recorded signal at the location $\vec{x}$ is first filtered on a bandwidth which includes the tones at the frequencies $n B \Omega_{r}$ (primary noise) and $n B\left(\Omega_{r}+\Omega_{\mathrm{o}}\right)$ (secondary source). The Hilbert transform modulus of the filtered signal is then computed, giving the total amplitude level $\left|p_{t}\left(\vec{x}, n B \Omega_{r}\right)\right|$. Since the obstruction has an angular periodicity of $2 \pi / n B$, the time segments of length $\Omega_{\mathrm{o}} /(n B)$ are averaged. The optimal angle $\phi_{\text {opt }}\left(n B \Omega_{r}\right)$ corresponds to the minimum of the mean total amplitude level. Note that since the obstruction is rotating, a further correction, $\Phi_{c}$, must account for the wake deviation induced by the rotation. It can be approximated by $\Phi_{c}=2 \pi \Omega_{\mathrm{o}} D / V_{\infty}$ with $D$ the distance between the obstruction and the rotor, and $V_{\infty}$ the mean upstream axial velocity. Step 4 is the validation of the optimal obstruction design. A last simulation with a static obstruction and the previous optimum parameters is achieved to confirm and estimate the expected noise reduction.

To perform this maximum of six simulations, the Lattice Boltzmann Method (LBM) has been selected [13, 14, 15], as it has been shown to accurately predict low-speed axial fan noise $[16,17,18]$. It could also yield similar accurate predictions for more complex axial fan systems $[19,20]$. Finally, the 


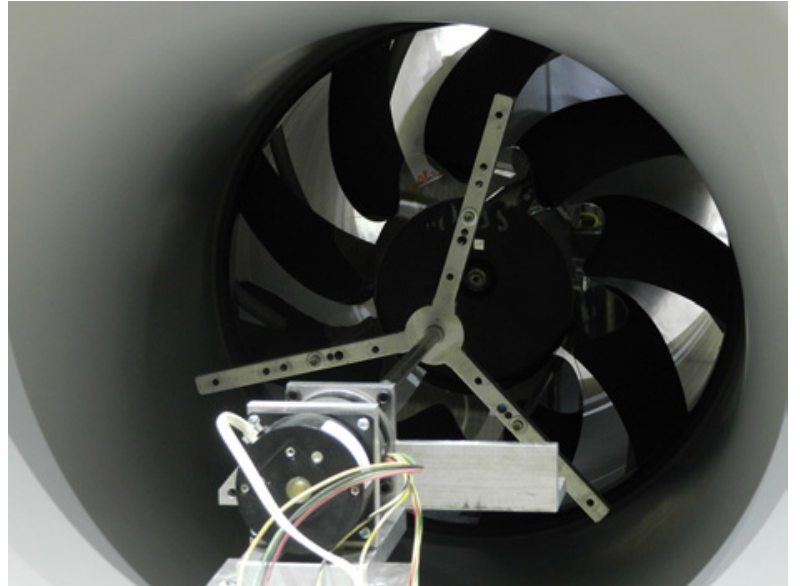

Figure 3. Controlled axial fan system with external obstruction.

ability of the method to predict noise control with static and rotating obstruction was recently demonstrated on a large industrial radial fan system [10].

\subsection{Experimental mock-up and test set-up}

The study of the obstruction-fan interaction has been carried out on the automotive cooling fan shown in Fig. 3, which has been extensively studied both experimentally and numerically $[21,17,18]$. The acoustic signature of the rotor alone was found to be dominated by a subharmonic tonal noise created by backflow vortices. Since this noise could not be controlled with a static obstruction, the radiation at the BPF has been strongly enhanced by adding a stator downstream of the rotor aligned with the rotor wake (Fig. 1). The fan system is placed in a short duct, and the operating point matches the design condition of the rotor $\left(2500 \mathrm{~m}^{3} / \mathrm{h}\right.$ and $2500 \mathrm{rpm}$ ).

The obstructions are then chosen to control the tonal radiation at the $\operatorname{BPF}(n=1)$, and the number of external lobes was equal to the number of rotor blades $(L=B=9)$. The inner radius corresponds to the rotor hub radius $\left(r_{1}=70 \mathrm{~mm}\right)$ and the thickness of the base $\left(r_{2}-A / 2-r_{1}\right)$ is set to $10 \mathrm{~mm}$. As explained above, the magnitude of the secondary source has then been adjusted by varying the lobe amplitude $A$, while the obstruction-fan distance remained constant $(50 \mathrm{~mm}$ between the obstruction and the rotor leading edge), large enough to neglect any potential effect of the rotor on the obstruction. The reference angular position $\phi=0^{\circ}$ is arbitrarily chosen when the obstruction lobes are aligned with the stator vanes at mid-lobe radius $r_{2}$.

The whole system has been tested in the hemi-anechoic room at UdeS as shown in Fig. 4, where the volume flow-rate imposed by the plug is measured simultaneously with sound pressure levels in the far-field. The flow rates have been estimated by integrating the velocity measured at the outlet plug with a Pitot tube. The far-field acoustic pressure has been measured $1.8 \mathrm{~m}$ away from the rotor center with eight 1/2-inch PCB HT378Bo2 microphones (377Bo2 microphone

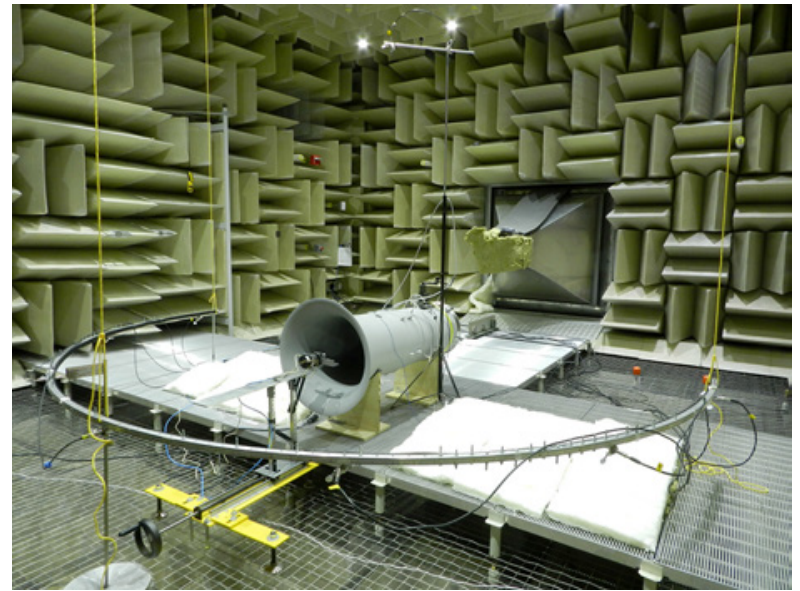

Figure 4. Experimental setup in the UdeS fully anechoic room.

and $\mathrm{HT}_{42}$ 2Eo1 preamplifier) to yield the module directivity as shown in Fig. 4. Five wall-pressure probes measure the static pressure inside the duct on a plane $150 \mathrm{~mm}$ downstream of the stator plane. Their values are averaged by connecting the probes to a single tube. The pressure rise across the fan is then determined by the pressure difference between the probes and the ambient static pressure in the anechoic room. Finally, a Brüel \& Kjaer (B\&K) accelerometer Type 4397 is positioned on the duct in order to measure its vibrations and verify that no significant acoustic radiation of the duct wall is measured by the microphones. The acquisition time was $40 \mathrm{~s}$ for all the measurements. The time history was recorded and the acoustic spectra were computed by the B\&K software PULSE with a Hanning windowing and a $75 \%$ overlap.

\subsection{Numerical set-ups and parameters}

The computational domain mimics the actual UdeS anechoic room by centering the short duct with the fan sytem in a large, 44-m long, cubic fluid domain. The computational domain is meshed using cubic voxels only, with eleven voxel refinement (VR) zones, the largest ones outside the testing region corresponding to the damping zones to reproduce the room anechoicity. Based on previous convergence studies on this fan, the smallest voxel has been set to $0.5 \mathrm{~mm}$ in a volume which includes the rotor, the stator and the obstruction [18]. A final mesh of 77 (respectively 81.5 ) million voxels is obtained for the fan system alone (respectively for the complete obstruction-fan system), which provides grid-independent acoustic solutions on the baseline configuration. As in the experimental tests, only the fan rotational speed is imposed to achieve the given operating point. The pressure rise and the flow rate result from the losses in the system and the plug set-up. An outlet boundary condition with a free flow direction and an imposed ambient static pressure is applied on all the faces of the simulation domain. The interface between the rotor part and the stationary part of the domain is a sliding mesh interface as explained in [16]. The Exa Powerflow 4.4 solver has been used to perform the compressible Very Large 


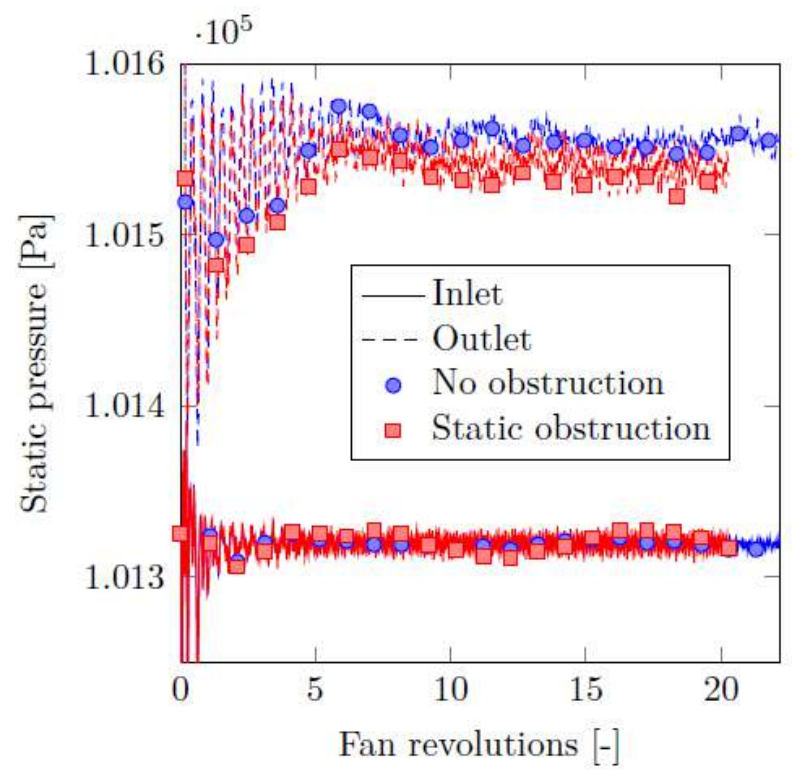

Figure 5. LBM convergence on static pressure.

Eddy Simulation (VLES) of this configuration.

The time step is selected by the solver to ensure a CFL number based on the speed of sound of one in the smallest voxels. This leads to a time-step of $8.2 \times 10^{-7} \mathrm{~s}$. The torque and the flow-rate are monitored during the simulation to evaluate the convergence of the system towards a steady state. Steady flow-rates on the inlets and outlets of the fan system were obtained after 2 fan revolutions for the reference configuration. For the obstruction case, converged flow conditions are obtained after ten fan revolutions, and stabilized results are even achieved after five revolutions as shown in Fig. 5. On the arc where the far-field acoustic pressure has been measured the voxel resolution is $64 \mathrm{~mm}$, which yields a direct accurate capture of the acoustic waves up to $1.2 \mathrm{kHz}$ or $3 \times \mathrm{BPF}$. In addition 62 point probes have been placed on a closer sphere of $1.5-\mathrm{m}$ radius from the rotor center to yield proper directivity and enough spatial resolution for sound power evaluation. The performance of the corresponding LBM simulations is summarized in Tab. 1.

Table 1. Performance of total noise predictions.

\begin{tabular}{c|c|c} 
LBM simulation & $\begin{array}{c}\text { Grid size } \\
\text { (million cells) }\end{array}$ & $\begin{array}{c}\text { Time/BPP } \\
\text { (for } 1 \text { core) }\end{array}$ \\
\hline Fan system & 69.1 & 12.7 days \\
\hline Fan system+obstruction & 81.5 & 13.8 days
\end{tabular}

\section{RESULTS AND DISCUSSION}

\subsection{Overall Fan Performance}

As mentioned above the present study first aims at performing a complete computation of the obstruction-fan inter- action to better understand the aeroacoustic mechanisms responsible for the tonal noise reduction.

The global performances of the installed fan (the pressure rise across the fan for a given mass-flow rate) are compared with the experimental measurements in Tab. 2. The numerical predictions are in good agreement with the experiment, showing a maximum of $4 \%$ difference with an obstruction lobe amplitude $A=30 \mathrm{~mm}$. This consistent 3-4\% discrepancy with and without obstructions is most likely caused by a slight lack of local grid resolution within the duct. Yet, the performance loss caused by the obstruction upstream of the fan is well predicted for both obstructions. These results confirm the relatively small influence of the obstruction on the fan performance and are coherent with the previously reported predictions $[8,22]$.

Table 2. Comparison between measured and computed pressure difference $[\mathrm{Pa}]$ (relative loss due to obstruction [\%]).

\begin{tabular}{c|c|c} 
& LBM Simulation & Experiment \\
\hline No obs. & $288(-)$ & $297.4(-)$ \\
\hline$A=20 \mathrm{~mm}$ & $279(3.1)$ & $287.1(3.4)$ \\
\hline$A=30 \mathrm{~mm}$ & $273(4.8)$ & $284.6(4.3)$
\end{tabular}

\subsection{Obstruction-Fan Interaction}

The flow topology and the wall-pressure fluctuations extracted from the computations provide important information which have never been captured by experimental studies.

\subsubsection{Flow topology}

The overall flow structure within the system is shown by the $\Lambda_{2}$ criterion in Fig. 6. Large backflow coherent vortices are observed near the rotor ring, as it was the case in the simulations without an obstruction $[21,18]$ or on modules for similar ring fans $[19,20]$. As for the obstruction wake, the simulation highlights vortex rings formed around the lobes

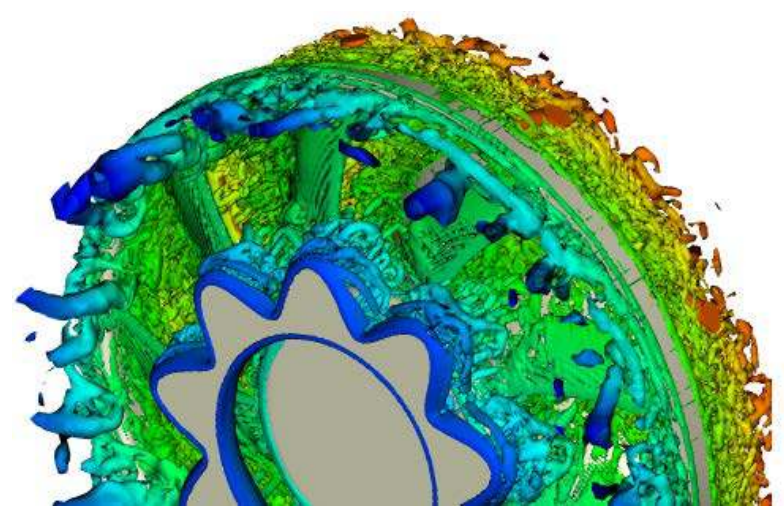

Figure 6. Simulated vortex structure with a static obstruction $(A=30 \mathrm{~mm}): \lambda_{2}$ iso-contours colored by the axial position. 


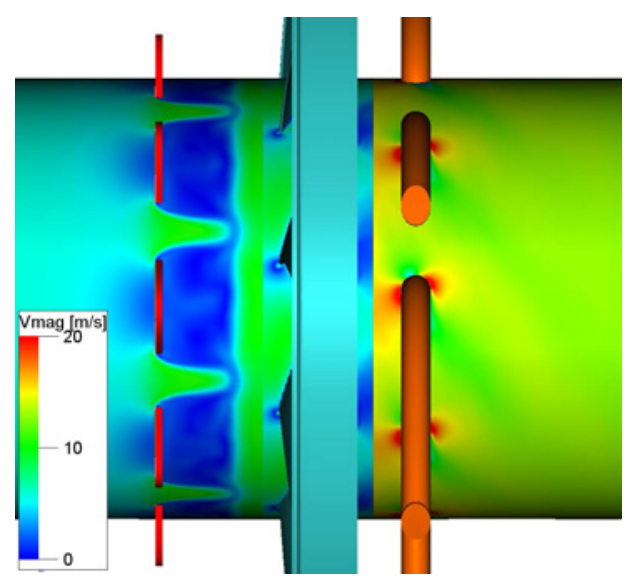

(a) With a static obstruction.

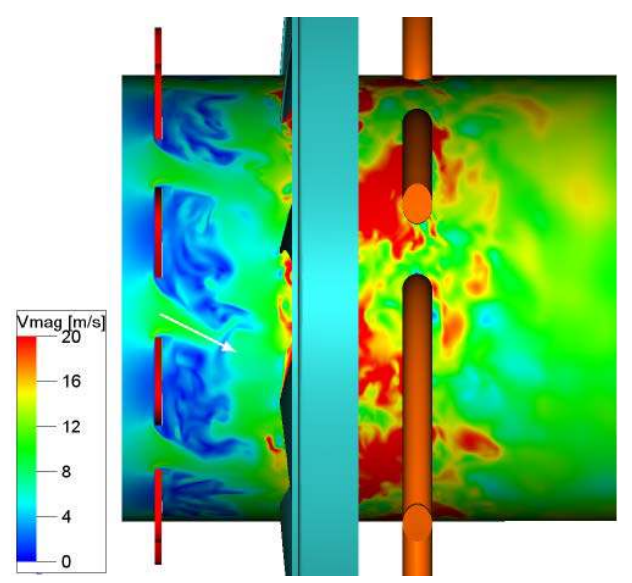

(b) With a rotating obstruction.

Figure 7. Velocity magnitude at mid-lobe radius $r_{2}=87.5$ $\mathrm{mm}(A=30 \mathrm{~mm})$.

and convected downstream to the fan blade. This aerodynamic phenomenon creates a velocity deficit downstream of the obstruction lobes and therefore generates a non-uniform circumferential velocity profile. Similarly to a stator-rotor interaction, this azimuthal non-uniformity induces a periodic fluctuation of the blade load and thus an additional tonal noise source.

This observation is confirmed by the velocity field at midlobe radius presented in Fig. 7 for a static and a rotating obstruction. A large velocity deficit is formed downstream of the obstruction lobe, which creates a non-uniform velocity profile in the rotor plane. Moreover, it should be noted that the obstruction rotation generates a deviation of the lobe wakes with respect to the rotating axis (see white arrow in Fig. 7 (b)). This confirms the need to correct the obstruction angular position when post-processing the acoustic data obtained with a rotating obstruction, as was proposed above (see the angle-correction $\Phi_{c}$ definition in Sec. 1.1).

The correction angle $\Phi_{c}$ was estimated in the simulations from the deviation angle for several obstruction rotational speeds $\Omega_{\mathrm{o}}$. Fig. 8 presents the results compared with the theoretical correction angle calculated in Sec. 1.1. An excellent agreement is found, confirming the validity of the angle correction introduced above.

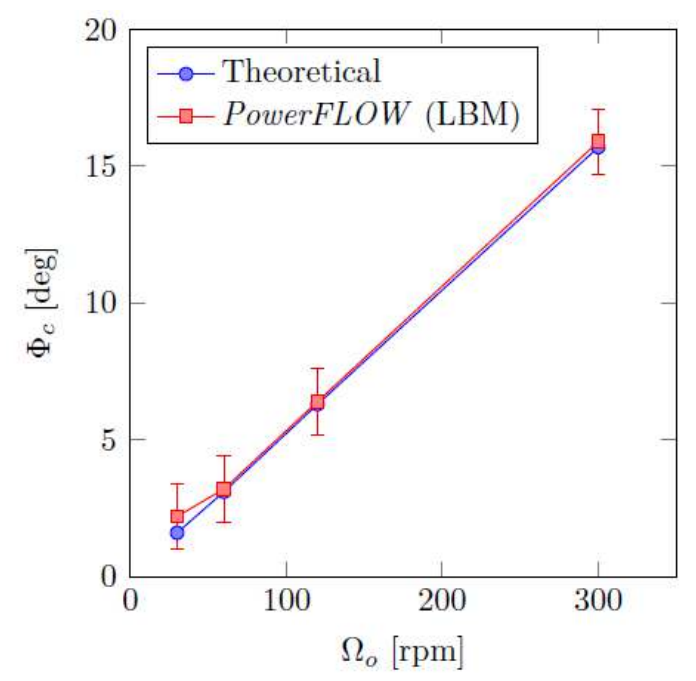

Figure 8. Correction angle $\Phi_{c}$ as a function of the obstruction rotational speed $\Omega_{\mathrm{o}}$.

\subsubsection{Acoustic source location}

A hint at the acoustic sources is provided by the zones of intense pressure fluctuations. The band-filtered power spectral density (PSD) of the wall-pressure fluctuations at the BPF is thus considered. Figs. 9 (a) and (b) show it for the fan system alone without obstruction on the suction side of the rotor and stator respectively. Other subfigures correspond to similar views of the same fan system with a static obstruction.

On the rotor with obstruction (Fig. 9 (c)), the high fluctuation spot at the blade-tip leading edge near the ring is similar to what can be observed with the rotor-stator alone (Fig. 9 (a)). However, an additional acoustic source is located in the lower half of the blade. This dominant source is generated by the interaction between the obstruction lobe wakes and the rotor.

On the stator with obstruction (Fig. 9 (d)), the sources are also affected when compared with the numerical results without an obstruction (Fig. 9 (b)). The same sources caused by the rotor wakes are found in the upper half of the vanes, but an additional source is present in the lower half. After being chopped by the rotor, the obstruction wakes are thus convected downstream to create a significant acoustic source on the stator.

The pressure fluctuations on the obstruction surfaces show much smaller amplitudes (Figs. 9 (e) and (f)), presumably created by the rotor potential effect on the downstream side of the obstruction. The noise sources located on the obstruction are negligible in comparison with the sources on the fan. Therefore, the obstructions are not only acoustically transparent (negligible diffraction), but they also do not generate significant noise themselves. The secondary noise source $\left(p_{s}\right)$ is clearly located on the rotor as a result of 


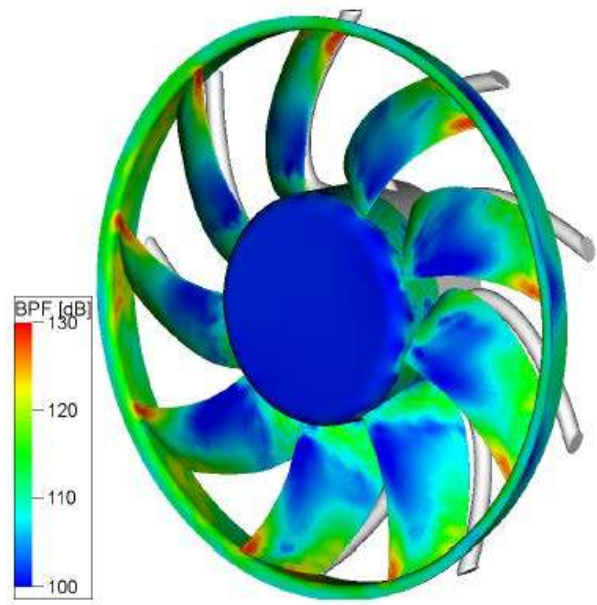

(a) Rotor without obstruction.

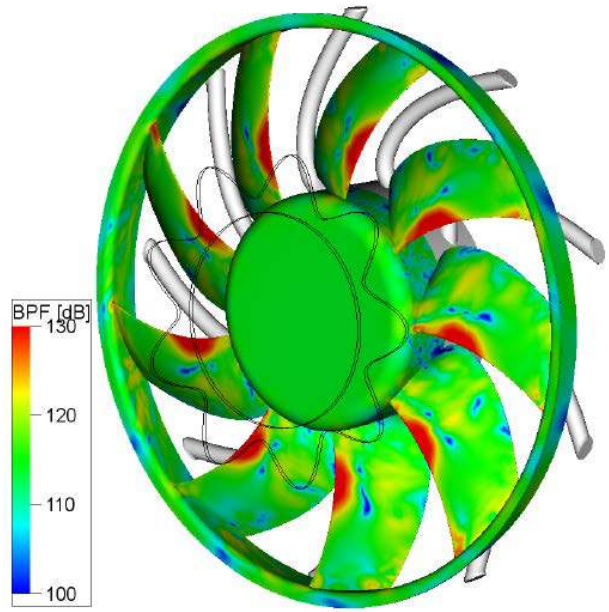

(c) Rotor with obstruction.

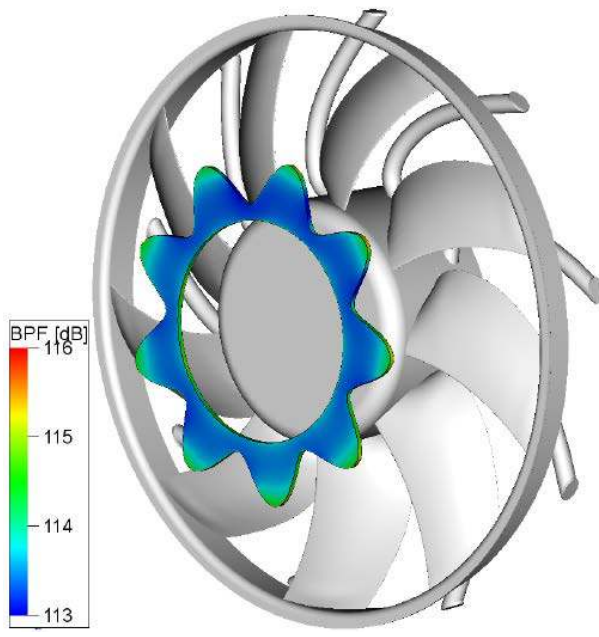

(e) Obstruction (upstream side).

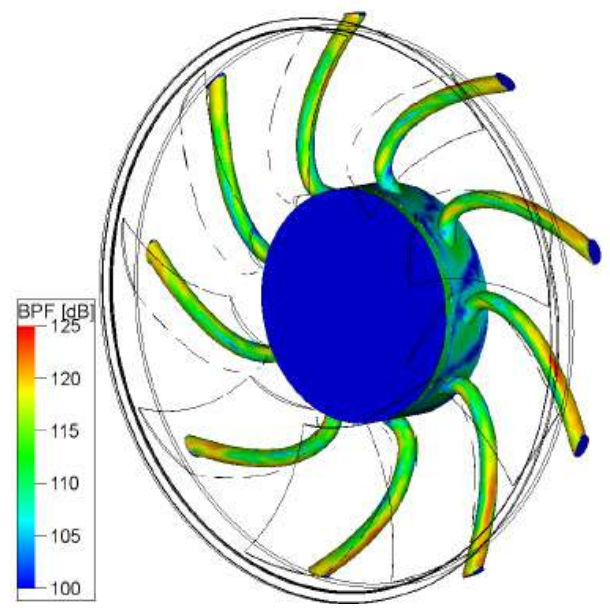

(b) Stator without obstruction.

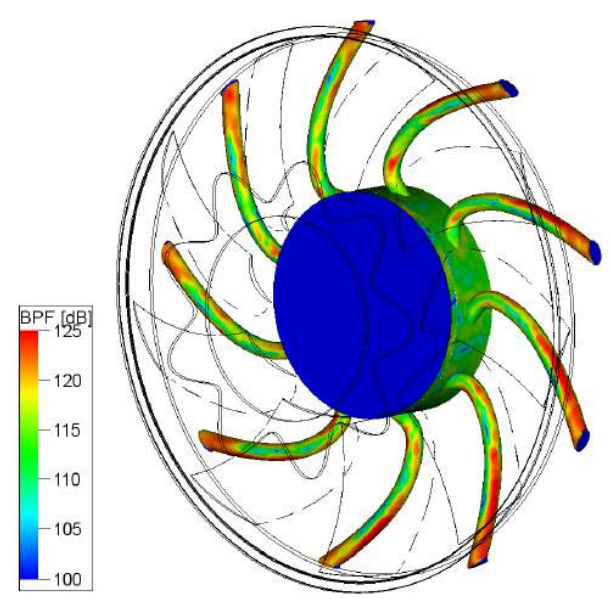

(d) Stator with obstruction

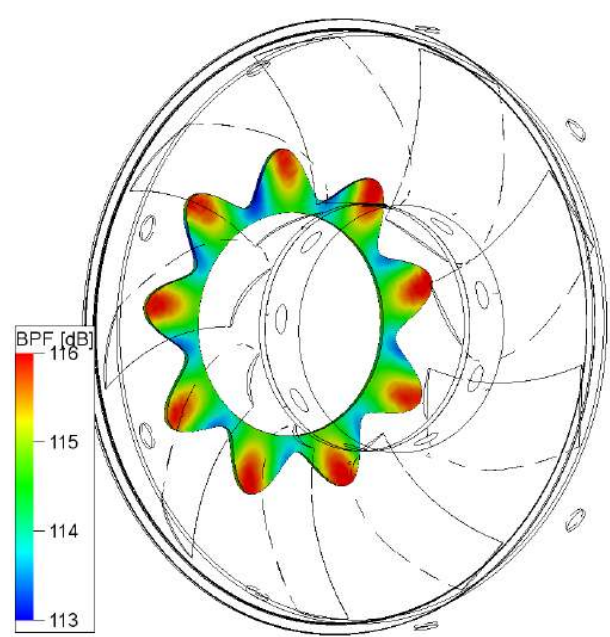

(f) Obstruction (downstream side).

Figure 9. Wall-pressure fluctuations at the BPF with a static obstruction. 
obstruction-wake interaction.

\subsection{Numerical Design of the Obstruction}

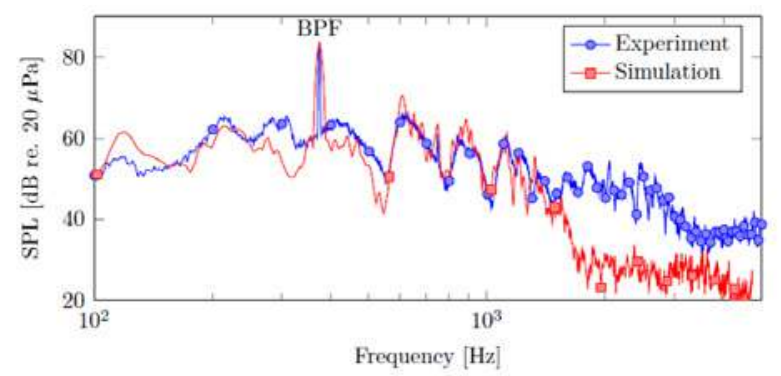

(a) Microphone on the rotor axis.

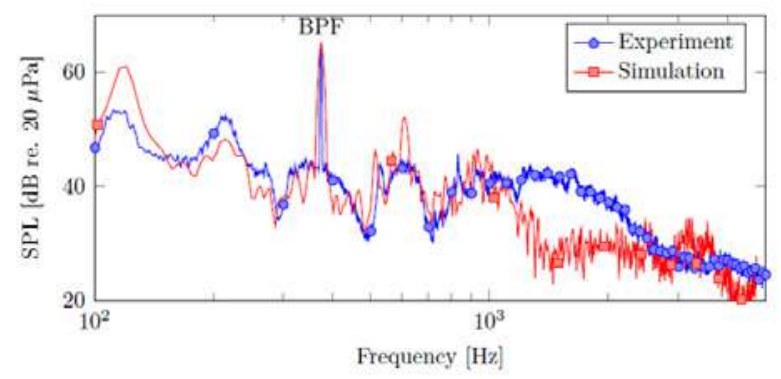

(b) Microphone in the rotor plane.

Figure 10. Comparison of sound pressure levels for the rotor-stator alone.

As mentioned in Sec. 1.1 the first step has consisted in validating and characterizing the primary source for the rotorstator alone $\left(p_{p}\right)$. As expected from the alignment of the cylindrical struts with the fan wake, a strong wake interaction yields strong wall-pressure fluctuations seen on the downstream stator as already shown in the case of static obstruction (Fig. 9 (b)), which in turn produces the strong tone at the BPF that can be seen in all directions (Fig. 10). The level of the latter is well captured by the simulation (less than $1 \mathrm{~dB}$ difference within the experimental uncertainty). This tone is also found to be very stationary in both experiment and simulation, which is a necessary assumption for a proper control. Moreover the broadband levels are also well captured by the direct LBM noise prediction, up to the local cut-off frequency of $1.2 \mathrm{KHz}$ of the Cartesian grid (Sec. 1.3).

Subsequently the optimal lobe amplitude is estimated by performing simulations with rotating obstructions. The first simulation was performed prior to the experiments with a lobe amplitude $A$ and an obstruction rotational speed $\Omega_{\mathrm{o}}$ arbitrarily set to $30 \mathrm{~mm}$ and $300 \mathrm{rpm}$, respectively. Fig. 11 shows the corresponding acoustic prediction. Comparing with the rotor-stator computation, this simulation shows a similar tone amplitude at the BPF and an additional tone at the frequency $\mathrm{BPF}+\mathrm{LPF}$ which corresponds to the secondary noise created by the rotating obstruction. In terms of levels, this secondary tone is higher than the BPF tone, which implies that the obstruction effect is too strong. A second

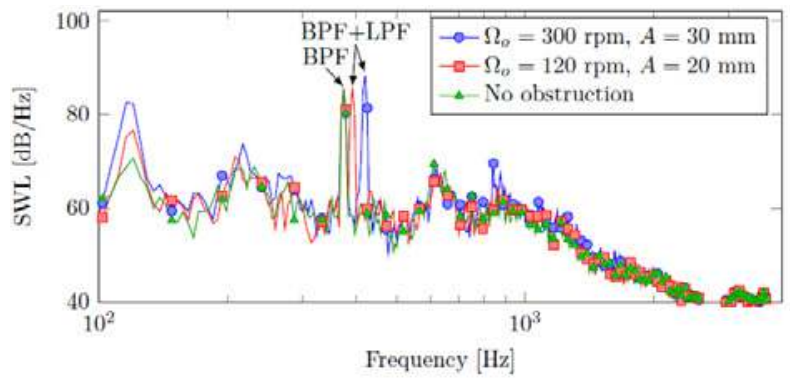

Figure 11. Comparisons of sound power levels for two simulations with a rotating obstruction and the simulation without obstruction.

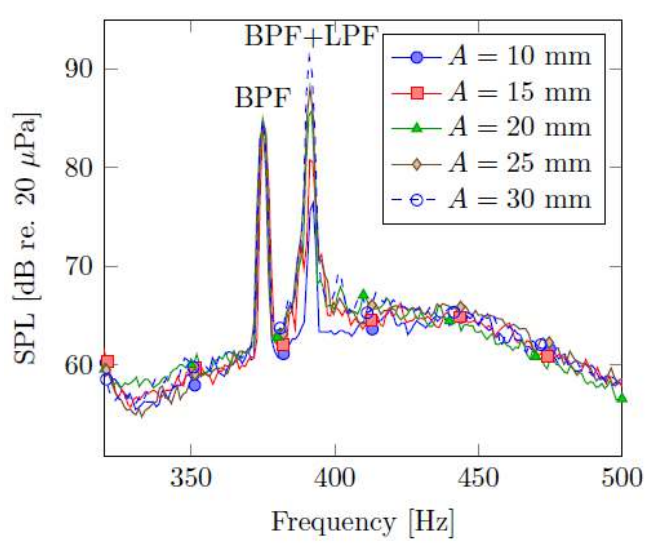

(a) Experimental sound pressure level $\left(\Omega_{\mathrm{o}}=120 \mathrm{rpm}\right)$.

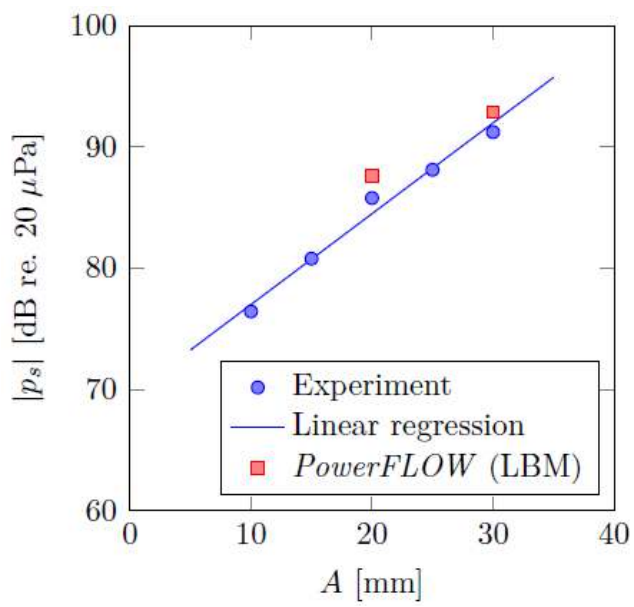

(b) Secondary source level as a function of the lobe amplitude.

Figure 12. Effect of the lobe amplitude with a rotating obstruction (microphone on the rotor axis, upstream of the fan).

simulation was thus performed with a smaller obstruction lobe amplitude $(A=20 \mathrm{~mm})$. The rotational speed was also changed $\left(\Omega_{\mathrm{o}}=120 \mathrm{rpm}\right)$ to evaluate its influence. In this configuration, the secondary tone occurred at a lower frequency due to the smaller rotational speed, and its level was found 


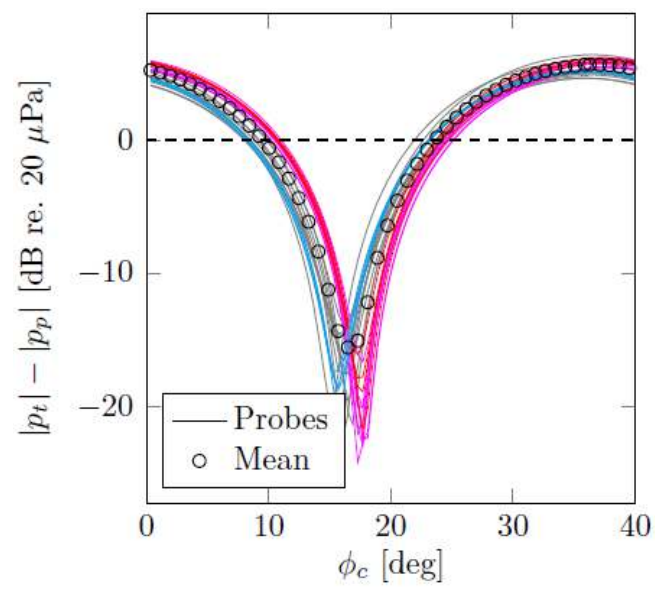

(a) $\Omega_{\mathrm{o}}=60 \mathrm{rpm}$.

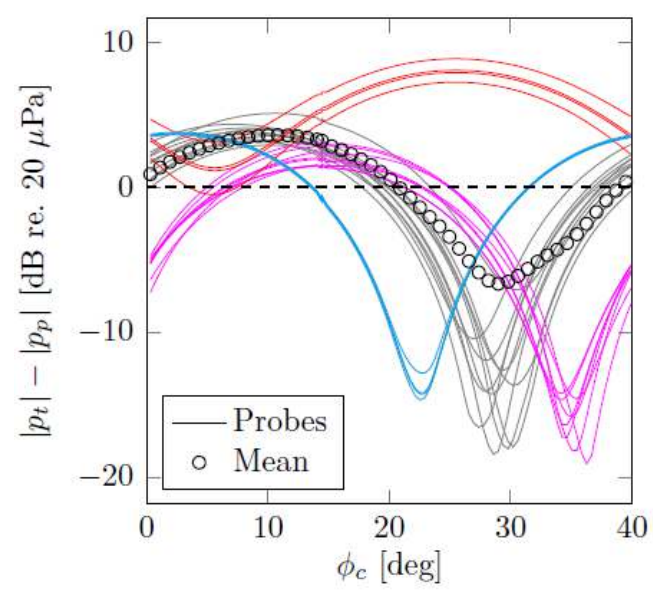

(b) $\Omega_{\mathrm{o}}=300 \mathrm{rpm}$.

Figure 13. Numerical amplification at the BPF as a function of the corrected angular position for all microphones.

to be the same as the level at the BPF (Fig. 11). Therefore, the optimal lobe amplitude predicted numerically is $A_{\text {opt }}=20$ $\mathrm{mm}$. Although the methodology in Sec. 1.1 indicates that three simulations are necessary to predict the optimal lobe amplitude, only two simulations suffice in the present study because of a serendipitous second choice of lobe amplitude. This optimal lobe amplitude was consequently verified experimentally by rotating several obstructions with different lobe amplitudes at $120 \mathrm{rpm}$. The results are shown in Fig. 12. First, it should be noted that the broadband noise level and the BPF amplitude are invariant (Fig. 12 (a)). This confirms the frequency selectivity of the obstruction. Secondly, the secondary source level varies linearly with the lobe amplitude (Fig. 12 (b)), which confirms another assumption of the methodology (see Sec. 1.1). Finally, the optimal lobe amplitude is found to be $A_{o p t}=20 \mathrm{~mm}$, which exactly matches the numerical prediction. The same results were found at the other microphone locations.

The third step consists in estimating the optimal angular position. From the numerical results with an obstruction in

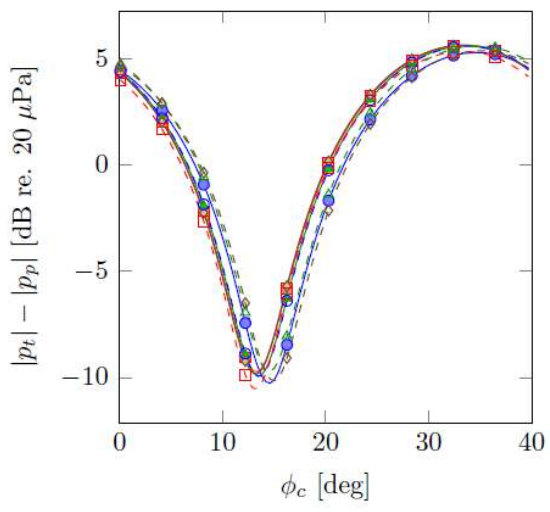

(a) $\Omega_{\mathrm{o}}=60 \mathrm{rpm}$.

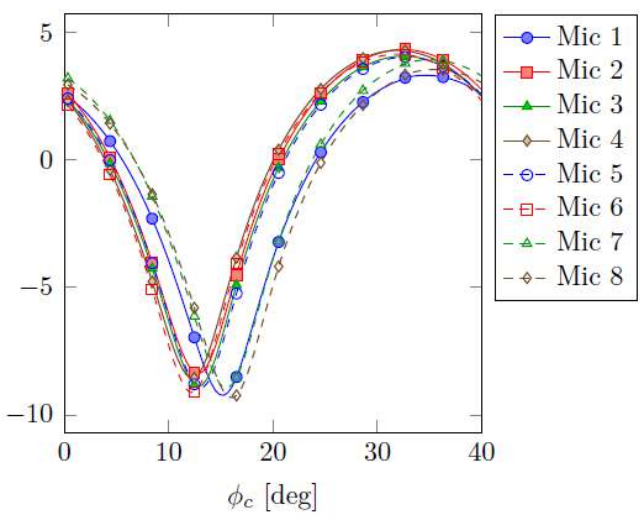

(b) $\Omega_{\mathrm{o}}=140 \mathrm{rpm}$.

Figure 14. Experimental amplification at the BPF as a function of the corrected angular position for all microphones.

rotation, the total amplitude level $\left|p_{t}\right|$ as a function of the angular position $\phi$ is extracted. Four different obstruction speeds have been simulated: $\Omega_{\mathrm{o}}=30,60,120$, and $300 \mathrm{rpm}$. Fig. 13 presents the BPF amplification or attenuation $\left(\left|p_{t}\right|-\right.$ $\left.\left|p_{p}\right|\right)$ as a function of the corrected angular position $\phi_{c}$ which takes into account the angle correction introduced in Sec. 1.1 for $\Omega_{\mathrm{o}}=60$ and $300 \mathrm{rpm}$. Groups of probes are highlighted in different colors to better demonstrate the directivity effect: the pink group corresponds to the plane of rotation, the blue and red ones to the downstream direction, and the grey ones to the upstream direction. A good agreement between all microphones is found for obstruction rotational speeds up to $\Omega_{\mathrm{o}}=60 \mathrm{rpm}$. Beyond, the greater the obstruction rotating speed is, the stronger the differences between the probes are, and the more difficult the optimal angle prediction is. Below $\Omega_{\mathrm{o}}=60 \mathrm{rpm}$, all the probes show a similar attenuation as a function of the obstruction angular position, yielding the optimal angle $\phi_{c, o p t}=16^{\circ}$. Note that the mean maximum attenuation is around $-15 \mathrm{~dB}$ with some substantial variations caused by the tone stability evidenced by looking at the temporal fluctuations of the Hilbert transform modulus.

In the corresponding measurements, five obstruction speeds $\Omega_{\mathrm{o}}$ have been tested: 6o, 80, 100, 120, and $140 \mathrm{rpm}$. 
Note that the step motor did not allow to go beyond $140 \mathrm{rpm}$. When the total amplitude levels as a function of the corrected angular position for a microphone in the rotor plane is plotted, similar behavior and shape of the BPF amplification or attenuation as in the simulations is observed (Fig. 14). Up to $\Omega_{\mathrm{o}}=6 \mathrm{o} \mathrm{rpm}$, all results collapse showing an optimal obstruction angular position $\phi_{c, o p t}$ of about $15 \pm 1^{\circ}$, very close to the above numerical prediction. The uncertainty is caused by the selected tachometer synchronization [23]. The maximum attenuation varies around $-10 \mathrm{~dB}$ lower than in the simulation most likely caused by the tone stability also found experimentally.

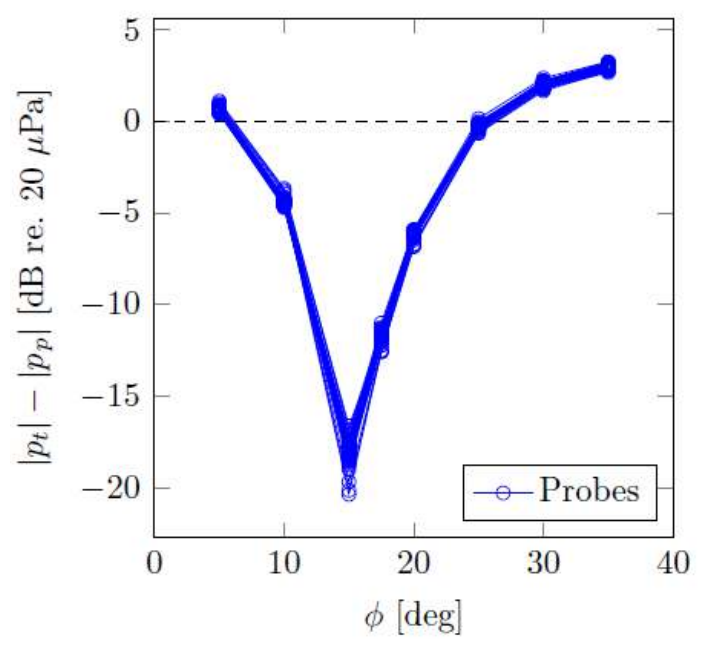

Figure 15. LBM results with a static obstruction: BPF amplification as a function of the obstruction angular position for all probe locations.

Finally the optimal design was checked with a static obstruction. The numerical result for several obtruction positions is shown for instance in Fig. 15. Both numerical and experimental results show an optimal position around $\phi_{c, o p t}=15^{\circ}$, which is in excellent agreement with the predictions in rotation ( $16^{\circ}$ for the LBM). The noise attenuation is identical for all microphones, which stresses that the obstruction does not show any significant directivity effect.

\section{CONCLUSION}

A complete investigation of the tonal noise control with flow obstruction of an axial low-speed fan system has been achieved both numerically and experimentally.

A direct acoustic propagation method using the LBM solver Powerflow 4.4 from Exa has been applied to investigate an axial low-speed fan system specifically designed to yield strong wake interaction and consequently tonal noise. The LBM simulation has first been shown to reproduce the aerodynamic performance and the acoustic far-field pressure of this fan system accurately. Noticeably the measured sound pressure levels are well reproduced at any microphone location, both in terms of levels and spectral shape for both tonal and broadband noise up to $1200 \mathrm{~Hz}$, the maximum fre- quency that can be directly resolved by the local voxel size at the microphone location. The same numerical set-up has then been used to study the obstruction-fan interaction in details and decipher the corresponding noise mechanism. The LBM simulations have highlighted vortex rings formed at the obstruction lobes that are convected downstream and impinge on the fan blades. These structures create an azimuthal variation of the velocity profile which generates a periodic fluctuation of the blade load. As a result, the main acoustic sources induced by the obstruction is located on the fan surfaces, and the noise radiation coming from the obstruction itself is negligible. The latter is also shown to be acoustically transparent.

An industrially-applicable numerical methodology has then been proposed to obtain the optimal obstruction design for a given fan geometry and operating condition, prior to any prototyping and measurement. It relies on a maximum of six simulations of the fan system without and with the obstruction being static and slowly rotating. The first simulation of the fan system alone provides the reference primary noise source to be controlled. In the optimization process, simulations of rotating obstructions allow the separation of the fan and obstruction contributions to the noise (primary and secondary noise). The proposed methodology has been validated by parallel experiments in the newly designed anechoic wind tunnel at UdeS. Two simulations with rotating obstructions have sufficed to estimate the optimal lobe amplitude $A_{\text {opt }}=20 \mathrm{~mm}$, which has then been confirmed experimentally. The influence of the obstruction rotational speed $\Omega_{\mathrm{o}}$ has also been studied both numerically and experimentally. Results showed that strong discrepancies between the microphones appear for obstruction speeds above $60 \mathrm{rpm}$. However, simulations at the slowest speeds $\left(\Omega_{\mathrm{o}}=30\right.$ and $60 \mathrm{rpm}$ ) were in excellent agreement, predicting an optimal obstruction angular position $\phi_{c, o p t}=16^{\circ}$ very close to the optimal value obtained with a static obstruction. All these results have been confirmed experimentally even though the measurements with a rotating obstruction have highlighted the high sensitivity of the reference angle detection $\left(\phi=0^{\circ}\right)$, and have suggested that an extra care was necessary to obtain a good estimation of the optimal angular position. Similar significant BPF attenuation have been obtained between the measurements and the simulations, the latter being also able to predict the tone stability and controllability. Both simulations and experiments have also confirmed two assumptions of the methodology: the frequency selectivity of the obstruction and the linear variation of the secondary source level with the lobe amplitude.

Overall, the present study lays the foundation for the development of industrial numerical tools to reduce tonal noise from low-speed fans.

\section{ACKNOWLEDGMENTS}

Computations were made on the supercomputer MammothMP2 from Universite de Sherbrooke, managed by Calcul Québec and Compute Canada. The operation of this super- 
computer is funded by the CFI, NanoQuébec, RMGA and FRQ-NT. The authors would like to thank F. Pérot from Exa for fruitful discussions and technical support. Finally they would like to acknowledge NSERC for funding this INNOV research project.

\section{REFERENCES}

[1] V. Kota and M. C. M. Wright. Wake generator control of inlet flow to cancel flow distortion noise. Journal of Sound and Vibration, 295(1-2):94-113, 2006.

[2] Kenneth A. Kousen and Joseph M. Verdon. Active control of wake/blade-row interaction noise. AIAA 7., 1994.

[3] Y. Pasco, T. Guédeney, A. Leung-Tack, A. Berry, Moreau S., and P. Masson. Active noise control simulation of tonal turbofan noise in aero engines. In $20^{\text {th }}$ AIAA/CEAS Aeroacoutics Conference, Atlanta, GA, 16-20 june, 2014.

[4] Stéphane Moreau, Paul Laffay, Alexandre Idier, and Noureddine Atalla. Several noise controls of the trailingedge noise of a Controlled-Diffusion airfoil. In 22nd AIAA/CEAS Aeroacoustics Conference, number 20162816, Lyon, 2016.

[5] Anthony Gérard, Stéphane Moreau, Alain Berry, and Patrice Masson. Design of multi-modal obstruction to control tonal fan noise using modulation principles. $\mathcal{F}$. Sound Vib., 356:34-47, May 2015.

[6] Anthony Gérard, Michel Besombes, Alain Berry, Patrice Masson, and Stéphane Moreau. Tonal noise control from centrifugal fans using flow control obstructions. Noise Control Eng. F., 61(4):381-388, May 2013.

[7] Anthony Gérard, Alain Berry, Patrice Masson, and Stéphane Moreau. Use of a beat effect for the automatic positioning of flow obstructions to control tonal fan noise: Theory and experiments. F. Sound Vib., 332(19):4450-4460, May 2013.

[8] Anthony Gérard, Alain Berry, Patrice Masson, and Yves Gervais. Experimental validation of tonal noise control from subsonic axial fans using flow control obstructions. f. Sound Vib., 321(1-2):8-25, 2009.

[9] F. Pérot, M.-S. Kim, V. Le Goff, X. Carniel, Y. Goth, and C. Chassaignon. Numerical optimization of the tonal noise of a backward centrifugal fan using a flow obstruction. Noise Control Eng. f., 61(3):307-319, 2013.

[10] M. Sanjosé and S. Moreau. Direct noise prediction and control of an installed large low-speed radial fan. Eur. 7. Mechanics B/Fluids, 61:235-243, 2017. accepted for publication.

[11] Anthony Gérard, Alain Berry, and Patrice Masson. Optimization problem for the automatic positioning of flow obstructions to control tonal fan noise. Fournal of the Acoustical Society of America, 123(5):3539, 2008.

[12] Anthony Gérard, Alain Berry, Patrice Masson, and Yves Gervais. Modelling of tonal noise control from subsonic axial fans using flow control obstructions. F. Sound Vib., 321(1-2):26-44, March 2009.

[13] H. Chen. Volumetric formulation of the lattice Boltzmann method for fluid dynamics: Basic concept. Physical Review E, 58(3):3955-3963, September 1998.

[14] S. Chen and G. D. Dooler. Lattice Boltzmann method for fluid flows. Ann. Rev. Fluid Mech., 30:329-364, 1998.

[15] H. Chen, S. A. Orszag, I. Staroselsky, and S. Succi. Expanded analogy between Boltzmann kinetic theory of fluids and turbulence. F. Fluid Mech., 519:301-314, November 2004.

[16] F. Pérot, M.-S. Kim, S. Moreau, M. Henner, and D. Neal. Direct aeroacoustics prediction of a low-speed axial fan. In 16th AIAA/CEAS Aeroacoustics Conference, AIAA20103887 paper, Stockholm, Sweden, June 2010.

[17] M. Sanjosé, D. Lallier-Daniels, and S. Moreau. Aeroacoustics Analysis of a Low Subsonic Axial Fan. In ASME Turbo Expo 2015 Conference, number ASME GT201512345, Montreal, Canada, June 15-19 2015.

[18] S. Moreau and M. Sanjosé. Sub-harmonic broadband humps and tip noise in low-speed ring fans. f. Acoust. Soc. Am., 139(1):118-127, 2016.

[19] M. Piellard, B. Coutty, V. Le Goff, F. Pérot, and V. Vidal. Direct Aeroacoustics Simulation of Automotive Cooling Fan System: Effect of Upstream Geometry on Broadband Noise. In AIAA-CEAS 2014, Atlanta, Georgia, USA, 2014.

[20] D. Lallier-Daniels, Sanjosé, S. Moreau, and M. Piellard. Aeroacoustic Study of a Ring-Shrouded Axial Fan Using Lattice-Boltzmann Simulations. Eur. F. Mechanics B/Fluids, 61:244-254, 2017.

[21] S. Magne, S. Moreau, and A. Berry. Subharmonic tonal noise from backflow vortices radiated by a low-speed ring fan in uniform inlet flow. 7. Acoust. Soc. Am., 137(1):228-237, 2015.

[22] Stéphan Magne, Marlène Sanjosé, Stéphane Moreau, Alain Berry, and Anthony Gérard. Tonal Noise Control of Centrifugal Fan Using Flow Obstructions - Experimental and Numerical Approaches. In 19th AIAA/CEAS Aeroacoustics Conference, pages 1-13, Berlin, 2013.

[23] S. Magne. Approche numérique du contrôle du bruit tonal des ventilateurs par obstruction de l'écoulement. $\mathrm{PhD}$ thesis, Université de Sherbrooke, 2015. 